\title{
ARTICLE OPEN \\ Compliant plant wearables for localized microclimate and plant growth monitoring
}

\author{
Joanna M. Nassar ${ }^{1}$, Sherjeel M. Khan $\mathbb{D}^{1}$, Diego Rosas Villalva ${ }^{1}$, Maha M. Nour ${ }^{1}$, Amani S. Almuslem ${ }^{1}$ and Muhammad M. Hussain $\mathbb{D}^{1}$
}

The microclimate surrounding a plant has major effect on its health and photosynthesis process, where certain plants struggle in suboptimal environmental conditions and unbalanced levels of humidity and temperature. The ability to remotely track and correlate the effect of local environmental conditions on the healthy growth of plants can have great impact for increasing survival rate of plants and augmenting agriculture output. This necessitates the widespread distribution of lightweight sensory devices on the surface of each plant. Using flexible and biocompatible materials coupled with a smart compact design for a low power and lightweight system, we develop widely deployed, autonomous, and compliant wearables for plants. The demonstrated wearables integrate temperature, humidity and strain sensors, and can be intimately deployed on the soft surface of any plant to remotely and continuously evaluate optimal growth settings. This is enabled through simultaneous detection of environmental conditions while quantitatively tracking the growth rate (viz. elongation). Finally, we establish a nature-inspired origami-assembled 3D-printed "PlantCopter", used as a launching platform for our plant wearable to enable widespread microclimate monitoring in large fields.

npj Flexible Electronics (2018)2:24; doi:10.1038/s41528-018-0039-8

\section{INTRODUCTION}

With increased global population, we are constantly challenged to meet the demand of food and to maintain a clean environment. Thus, increasing agricultural output from harsh environments, infertile lands, and forests has become a necessity. Recent developments in the area of information and communication enabled the emergence of a new paradigm of ubiquitous and smart sensor technology that can be widely used to monitor and to control important environmental and botanical aspects of plants, water savings, and such. A number of abiotic factors such as drought, salinity, excess irrigation, and extreme temperature and humidity levels, can have huge impacts on crop productivity and survival rate of the plants. $^{1-5}$ Consequently, horticultural production has become more and more automated, and careful assessment of the variety of these factors in relation to growth rate is necessary to ensure healthy growth of plants. Presently, in a greenhouse or a crop field, centralized climate monitoring devices are used to monitor the surrounding climate conditions, ${ }^{6,7}$ and remotely acquired data are then used to either alter the environmental conditions if necessary, or solely used as a monitoring tool. However, those sensors fail to see the conditions experienced by each plant as the environmental conditions can change from one end of a greenhouse (or field) to another, leading to yield reduction because of varying conditions across the field. Other commercial options include baton-shaped sensory devices fixed in the soil, and aim at wirelessly monitoring moisture, soil quality, and temperature, such as PlantLink, ${ }^{6}$ Parrot Power Flower, ${ }^{7}$ and Parrot Pot. ${ }^{8}$ However, they are costly, uncontrolled for localized microclimate evaluation, and possess limited potential for use in large fields or greenhouses, where augmented local control is vital on a large scale.
Besides, there is no accessible solution in the market that allows us to monitor growth of plants. Quantitative monitoring of plant growth is of great importance to understand how ambient conditions are affecting healthy growth. Factors such as inadequate temperature levels and reduced moisture can obstruct the healthy development of a plant. To date, the only reliable way to monitor physical plant growth is through active imaging systems and dynamic user interfaces, using advanced technologies and equipment which allow the assortment of large and reliable data sets related to plant growth variables, such as daily shoot growth. ${ }^{9-11}$ Other techniques try to predict and optimize plant growth conditions by adjusting major growth parameters including environmental temperature, humidity, soil water, and illumination data. ${ }^{12}$ However, they do not reflect definite information from the plant itself. Very few findings exist to monitor physical plant growth. More recently, a study attempted at monitoring plant growth by using chitosan-based water ink to print strain sensors on the plants' already grown fruits/seeds. ${ }^{13}$ While this method can measure the overall expansion of exclusively the fruits, it does not provide concrete information about the growth of the plant. Although the printed sensor provides great adhesion with the fruit, the measured resistance is affected by general expansion of the seed and hence there is no control on identifying what growth is. Also, this latter method does not represent a universal solution to monitor plant elongation, as it requires local stretching of the stem internode (which is only found in monocots), whereas the majority of plant stems grow from the tip or apical meristem. ${ }^{14}$

This suggests a persistent need toward the development of devices that can quantitatively and remotely monitor plant growth and the localized microclimate conditions surrounding

\footnotetext{
${ }^{1} \mathrm{mmh}$ Labs, Electrical Engineering, Computer Electrical Mathematical Science and Engineering Division, King Abdullah University of Science and Technology (KAUST), Thuwal 23955-6900, Saudi Arabia

Correspondence: Muhammad M. Hussain (muhammadmustafa.hussain@kaust.edu.sa)

These authors contributed equally: Joanna M. Nassar, Sherjeel M. Khan.
}

Received: 22 December 2017 Revised: 31 July 2018 Accepted: 6 August 2018

Published online: 10 September 2018 
each plant. This entails the integration of sensors on the individual leaves and stems of the plant. Sensors should be sufficiently small, compliant, and light enough to be mounted on the leaves without harming the plant, underlining the need for flexible and seamless sensors. Using flexible, stretchable, and biocompatible materials, we design compliant plant wearables by integrating temperature, humidity, and strain sensors. The seamless environmental multisensory platform is ultra-light and effortlessly adapts to the irregular surface of the leaves, providing the necessary localized study of the surrounding microclimate. Meanwhile, packaged stretchable strain sensors, designed to be placed along the stem and internodes of the plant, provide a universal and controlled solution to quantitatively monitor plant elongation/growth. For a completely autonomous and remote plant wearable, we use design strategies to achieve a compact and low-power system. Plant sensors are integrated with a miniaturized programmablesystem-on-chip (PSoC) and a small rechargeable battery to realize a lightweight system using low-power Bluetooth technology, enabling interface to the cloud and continuous monitoring of realtime conditions.

\section{COMPLIANT AND BIOCOMPATIBLE PLANT SENSORS}

To quantitatively monitor plant growth through elongation, the stretchable strain sensor design was tailored in such a way to provide high sensitivity to micrometer-level length variations, while retaining enough stretchability to be anchored in any desired position on the plant. The challenge is to make the sensor light in weight, enough not to bend the branches of the plant and prevent any induced bending/fractures. The sensor needs to be stretchable yet highly sensitive to strain, thus we chose an intrinsically stretchable substrate material, polydimethylsiloxane (PDMS), on top of which we deposit a thin gold metal film as our strain-sensing material (Fig. 1). Since metals have a high Young's modulus, the metal would normally break after couple of $\%$ of tensile loading, leading to a discontinuity in the microstructure of the film. It was found that the failure strain of a gold wire with $500 \mathrm{~nm}$ thickness and $100 \mu \mathrm{m}$ width is about $5 \%$ and the strain-stress curve becomes flat after $2 \%{ }^{15}$ To overcome this challenge, we implemented the buckling technique which consists of pre-straining the elastomer, and then depositing the metal on top of it. A wrinkly metal wire has good stretchability by extending its wavy structure in a non-coplanar plane. Detailed schematics of the fabrication process flow is illustrated in Fig. $1 \mathrm{a}-\mathrm{e}$, and design rules are discussed in the "Materials and Methods" section. Note that resistance measurements vary with moisture content, hence the complete encapsulation of our sensor in a hydrophobic material, such as PDMS, enabled us to eradicate this unwanted effect that might lead to undesirable data variations. The final structure of the packaged stretchable sensor is shown in Fig. 1f, g, highlighting its thin and intrinsically stretchable nature, allowing it to uniformly adhere to plants while being ultra-lightweight.

On the other hand, for microclimate monitoring, we fabricate a flexible multisensory platform integrating both temperature and humidity sensors on the same flexible and ultra-light butterfly shaped polyimide (PI)/PDMS platform. Polymers are appealing because they exhibit long-term stability, are sensitive to high humidity, have low manufacturing costs, and can be easily included in an integrated circuit (IC) process. ${ }^{16,17} \mathrm{~A}$ polymer material that has high thermal stability and low dielectric constant is preferable. ${ }^{16}$ Polyimide has superior characteristics such as high temperature resistance, a low dielectric constant, good mechanical strength, and dimensional stability, and is typically the material of choice for a capacitive humidity sensor's dielectric, due to its higher affinity toward moisture absorption and desorption. ${ }^{16,18}$ PDMS layer was also used as a back support for our platform and a barrier from plant transpiration due to its hydrophobic nature. Detailed schematics of the fabrication process flow is illustrated in Fig. 1h, and design structures are discussed in the "Materials and Methods" section. The result is an ultra-light butterfly shaped multisensory platform that can be intimately placed of the plant leaf (Fig. 1i), and conforms to microlevel irregularities found on the surface (Fig. 2a-c). Plant leaves generally display irregular patterns (Fig. 2c), so it is essential that our sensory platform is thin and flexible enough to adapt with the uneven surface of the leaves, providing desired adhesion to allow robust placement and data collection.

\section{LOW POWER AND COMPACT SYSTEM DEVELOPMENT}

To achieve the goal of an autonomous plant monitoring device which is compact and lightweight, we simplified the system components to reduce both weight and footprint while maintaining necessary performance. We use Cypress $^{\circledR}$ (CyBle-222005) $10 \mathrm{~mm} \times 10 \mathrm{~mm} \times 18 \mathrm{~mm}$ Programmable System-on-Chip (PSoC) with integrated low-power Bluetooth transceiver, allowing wireless data communication to a smartphone, while data can be continuously logged and stored inside the PSoC's internal flash memory of $256 \mathrm{kB}$. There are two sensing components for the electronic interface: capacitance and resistance. Usually a capacitance-to-digital convertor IC is required to convert the value of capacitance into a digital number so the microprocessor can read the values. This consumes additional power and adds bulk to the system. We took minimalist approach to use the CapSense block in the PSoC (Fig. 2d) (originally used to detect finger touches) to measure the capacitance directly from the sensor without using any additional components. In case of our resistive sensors, generally a Wheatstone bridge is used to convert the resistance into voltage so that the microprocessor can read the voltage and convert to temperature. Wheatstone bridge contains four resistors, which consume additional large amount of current and space. To keep things simple, we configured the PSoC to form a current source inside the PSoC. This current source feeds a fixed amount of current into the resistive sensors and measures the output voltage, respectively. For minimal power consumption, we only fed a $600 \mu \mathrm{A}$ current into the resistive sensor for a power saving of about $99 \%$ compared to Wheatstone bridge, which would consume $470 \mathrm{~mA}$ current for a setup to read from our temperature sensor. The block diagram of the system interface is depicted in Fig. 2d, displaying simultaneous logging of data from both capacitive (for humidity sensor) and resistive (for temperature and strain) sensors through voltage readout.

The system is powered using a small rechargeable $3.7 \mathrm{~V} \mathrm{Li} \mathrm{lon}$ battery of small size $29 \mathrm{~mm} \times 36 \mathrm{~mm} \times 4.75 \mathrm{~mm}$, with a capacity of $500 \mathrm{mAh}$. The average lifetime of our wearable system is about 151 days at a logging rate of $2 \mathrm{~s}$, considering the system will be in low-power state at most times and get into active power mode every $2 \mathrm{~s}$ to gather data. The output from the system for capacitive readout using PSoC is in the form of a "Raw Output Value". An increase in capacitance linearly changes the raw output value in the PSoC. Therefore, as higher humidity levels are detected, an increase in the capacitance of the structure is expected, which will be reflected by an increase in the raw output value collected by our system. To convert the raw output value to its corresponding humidity percentage, we calibrated our system using a commercial humidity sensor (Sensirion ${ }^{\circledR}$ Digital Humidity Sensor SHTW2). As for the resistive output, we are simply detecting voltage, which is quite straightforward for strain sensing. However, in the case of temperature sensing, we wanted to make a sensitivity plot relating voltage output to temperature values, as represented by Fig. S1a, which shows a linear behavior with $0.96 \mathrm{mV} /{ }^{\circ} \mathrm{C}$ sensitivity for a wide temperature range from 25 up to $100^{\circ} \mathrm{C}$. Meanwhile, Fig. S1b highlights real-time voltage response from our system corresponding to the continuous changes in temperature. Temperature profile was collected separately from a commercial temperature 


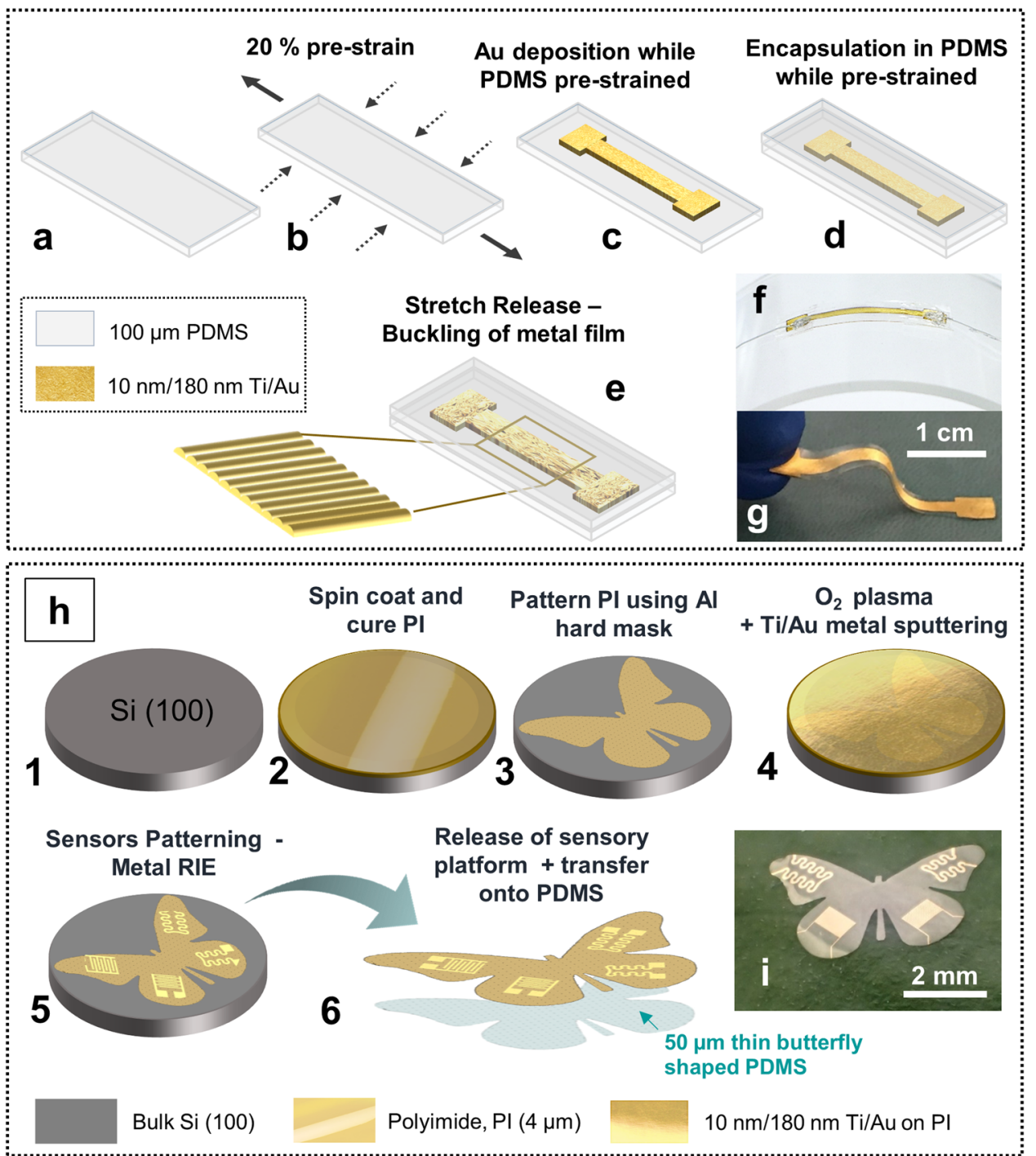

Fig. 1 Plant sensors design. Stretchable packaged strain sensors. a-e Schematic of the strain sensor's fabrication process flow, highlighting the buckled thin metal film structure. $\mathbf{f}$, $\mathbf{g}$ Digital photos highlighting the flexible, thin, and stretchable properties of the packaged strain sensor. $\mathbf{h}$ Flexible ultra-light sensors for microclimate detection. 1-5 Detailed schematic of the integration process of flexible and lightweight temperature and humidity sensors, and 6 final release of final butterfly sensory platform through simple peel-off, then transfer onto a thin layer of PDMS for a one-sided packaging. i Digital photograph of the compliant butterfly platform integrating environmental plant sensors and conformally placed on top of a plant leaf

sensor (Sensirion Digital Humidity Sensor SHTW2). Using the same parameters of the experiment, we then measure the temperature response of our integrated plant system, which is reflected through the voltage plant sensor profile in Fig. S1b. Both voltage and temperature profiles follow the same curves, highlighting reliable response and recovery behavior of our compact system, and consistency with commercial state-of-art sensing standards.

\section{RESULTS AND DISCUSSION}

Performance of the sensors

In strain, sensor is tested using an automated customized cycling setup which can be repeatedly controlled to get quantized amounts of tensile and compressive stretching, depicted in Fig. $3 a-c$ and demonstrated in Movie S1. Each stretching step corresponds to an elongation of $\Delta L=250 \mu \mathrm{m}$, for which we retrieve the corresponding resistance to get the resistance plot in Fig. 3d. The corresponding applied strain is calculated through the following relationship:

$\varepsilon=\frac{\left(L-L_{0}\right)}{L_{0}}$

Where $\varepsilon$ is the applied strain, " $L_{0}$ " is the initial length of the sensor, and " $L$ " is the final length of the sensor where $L=L_{0}+\Delta L$. Figure $3 \mathrm{~d}$ shows that our sensor can withstand up to $\sim 35 \%$ strain, while it displays a linear relationship up to $22 \%$ strain, which will be the preferable regime of operation. This $22 \%$ strain corresponds to an elongation of $\Delta L=3.75 \mathrm{~mm}$, which is more than enough to quantitatively and precisely monitor micrometer-level length growth of the plant through a given day. From the slope $\left(\frac{\Delta R}{\varepsilon}\right)$ of the plot in Fig. 3e, we can retrieve the gauge factor (GF), which is a more standard measure of strain sensitivity:

$\mathrm{GF}=\frac{\Delta R}{R_{0}} / \frac{\Delta L}{L_{0}}=\frac{\Delta R}{R_{0}} / \varepsilon=\frac{\Delta R}{\varepsilon} / R_{0}$

Accordingly, we calculate on average a gauge factor of GF $=3.9$ in the linear regime with stretchability of up to $22 \%$ strain. Generally, high-gauge factors are difficult to achieve while 

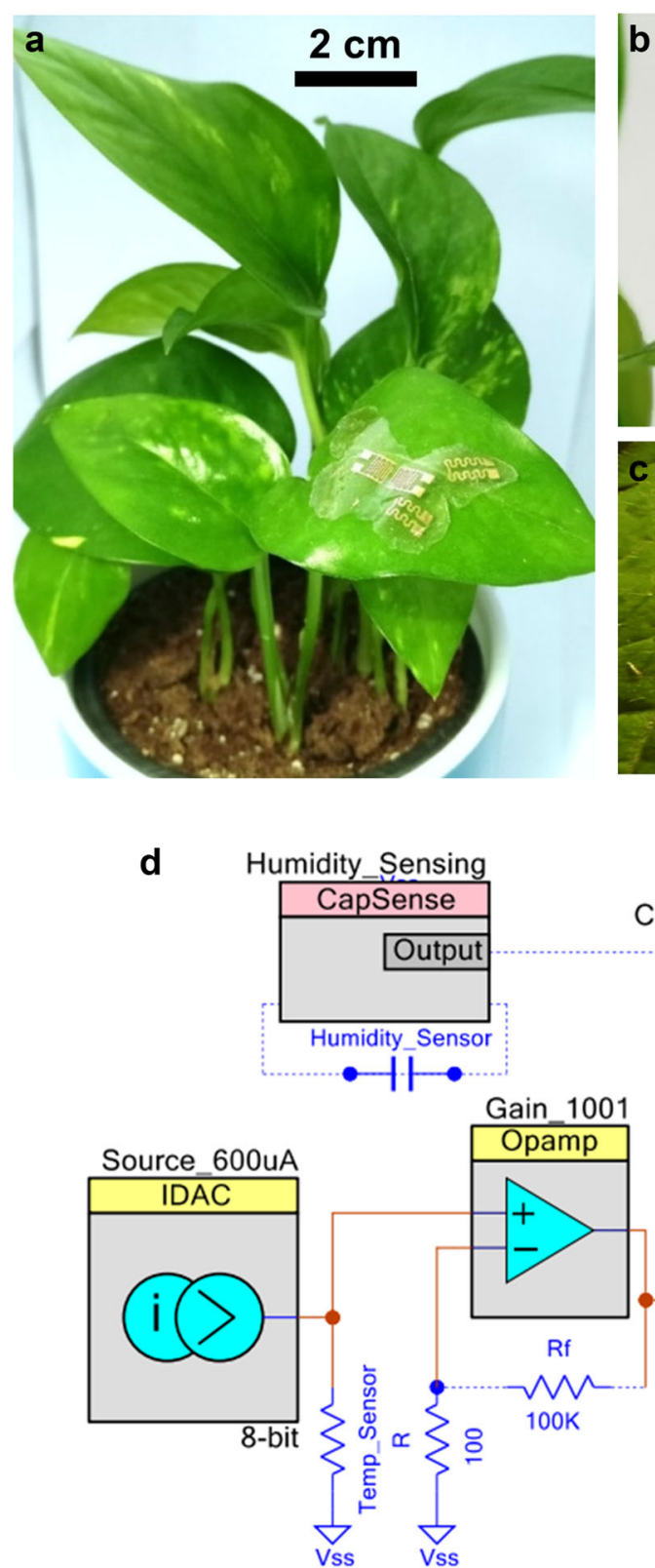
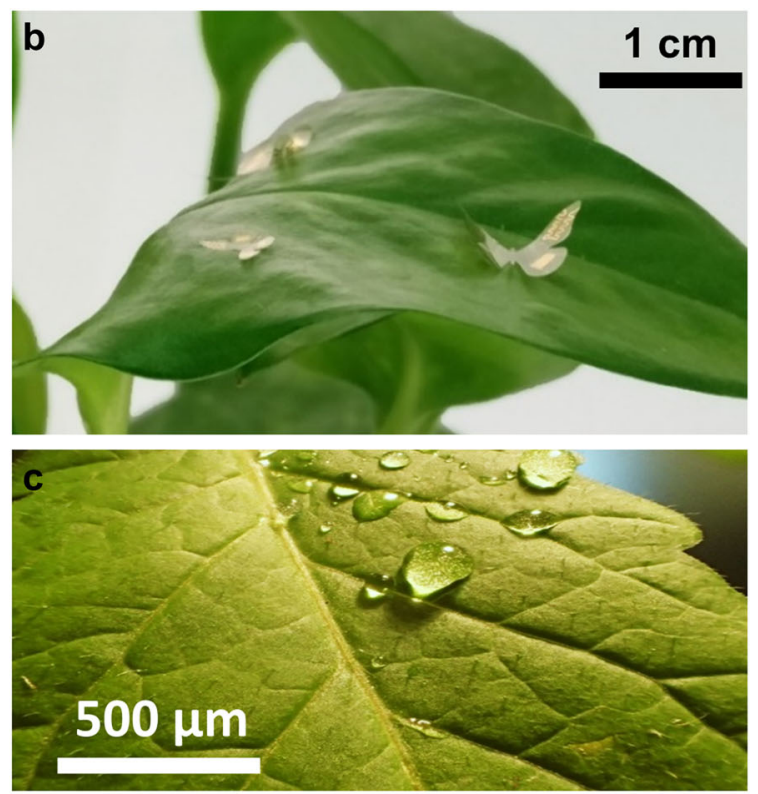

Data Save

Flash Memory
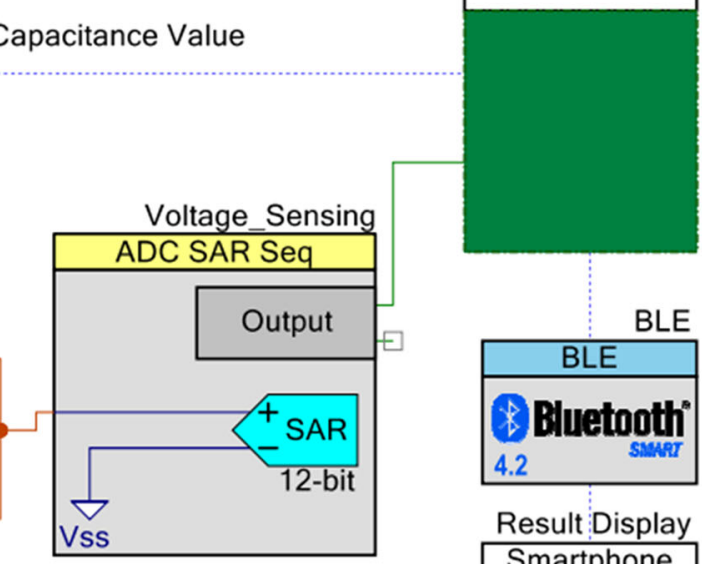

Result Display

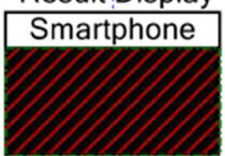

Fig. 2 Conformal integration of plant sensors. a Digital picture highlighting the intimate adhesion and conformal placement of environmental plant sensors platform on the surface of a plant leaf. b Digital photograph depicting the lightweight characteristic of the butterfly shaped sensory platform. c Zoomed-in photograph underlining the irregularities found on the surface of a plant leaf. d Block diagram of system components for plant sensors monitoring device, illustrating integration with a PSoC module for both resistive (voltage sensing) and capacitive sensing

retaining high stretchabilities for thin film metals. Conventional thin film metal strain sensors display $\mathrm{GF} \sim 2$ with maximum stretchability of $5 \% .{ }^{19}$ Sensors with GF above 200 are often limited by a stretchability of $5 \% .{ }^{20,21}$ Other highly stretchable strain sensors based on CNT networks show a GF $=0.82$ for stretchabilities from $0 \%$ up to $40 \%$ strain $^{22}$ and often suffer from nonlinearity and high hysteresis, ${ }^{23}$ while a more recent study on monitoring fruit growth using ink-based stretchable strain sensors displayed a gauge factor of $\mathrm{GF}=64{ }^{13}$ but limited in terms of practicality and linearity up to only $8 \%$ strain. Therefore, a balance between sensitivity and desired stretchability needs to be attained depending on the application of interest. In this work, by using the buckling technique, we were able to achieve higher stretchabilities using conventional thin film metal strain sensors. The achieved sensitivity of GF $=3.9$ is high enough to differentiate micrometer elongations in the plant's growth, while maintaining great stability and linearity up to $22 \%$ strain. We also explore the repeatability of the sensor by exposing the sensor to 500 cycles of stretch/release, automatically controlled through a smartphone application (Fig. $3 f$ for precise and repeatable cycles, as shown in Movie S2. Each stretching step is characterized by a $5.88 \%$ tensile strain, which translates into a $1 \mathrm{~mm}$ elongation. The cycling setup is depicted in Fig. 3a, c, and results for the first 10 cycles are shown in Fig. $3 \mathrm{~g}$, highlighting the consistent behavior of the sensor as it keeps recovering back to its initial resistance repetitively, with no hysteresis.

Temperature sensor's sensitivity plot (Fig. 3h) displays an expected linear increase in resistance with respect to higher temperatures, due to higher phonon vibrations and collisions. We measure a sensitivity of $0.032 \Omega /{ }^{\circ} \mathrm{C}$ and a corresponding 

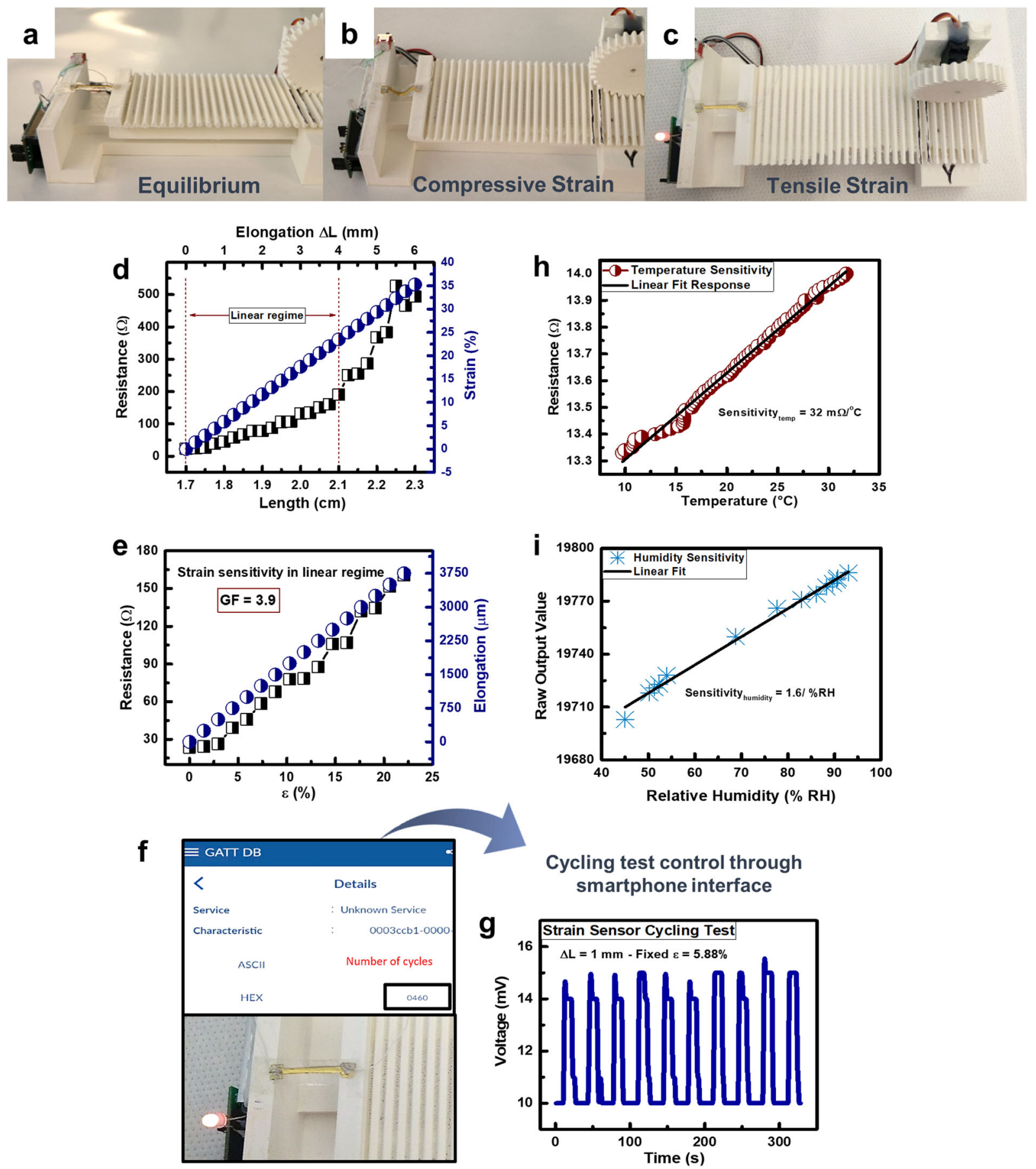

Fig. 3 Performance characteristics of plant sensors. a-c Digital photographs demonstrating our 3D-printed cycling setup used to perform strain tests, depicting the mounted sensor is under equilibrium, compressive, and tensile strain. $\mathbf{d}$ Plot showing the resistance increase as the strain sensor is stretched and elongated in a tensile manner, with a corresponding plot (in blue) relating elongation to tensile strain induced on the sensor. e Strain sensor sensitivity plot displaying a linear behavior up to $22 \%$ strain applied, corresponding to $3.75 \mathrm{~mm}$ elongation in the sensor's structure. $\mathbf{f}$ Photograph of the smartphone app interface for our automated strain cycling setup, reflecting experimental results for 500 cycles. Remote connection is performed via Bluetooth, and real-time changes in resistance values are reflected through changes in the "HEX" number seen in the black box. $\mathbf{g}$ Real-time plot of stretch/release cycles of strain sensors elongated by $1 \mathrm{~mm}(\varepsilon=5.88 \%)$. $\mathbf{h}$ Temperature sensitivity plot showing linear response. i Humidity sensitivity plot showing a quite linear response up to $93 \% \mathrm{RH}$

temperature coefficient of resistance of $\mathrm{TCR}_{\mathrm{Au} \text {,thin film }}=0.0024 /{ }^{\circ} \mathrm{C}$, which is slightly lower than the theoretical TCR value of bulk $\mathrm{Au}$ metal $\left(\mathrm{TCR}_{\mathrm{Au}}\right.$, bulk $\left.=0.0034 /{ }^{\circ} \mathrm{C}\right)$, and is expected due to increased porosity of sputtered thin film metals compared to bulk. Similarly, the performance of humidity plant sensor was tested by exposing the sensor to relative humidity levels from $45 \% \mathrm{RH}$ up to $93 \% \mathrm{RH}$, at room temperature. Figure $3 \mathrm{i}$ shows a linear response of sensitivity humidity $=1.6 / \% \mathrm{RH}$ and a linearity of 0.996 . Response to humidity changes was collected using our autonomous integrated system, hence the measured "Raw output value". This raw output 


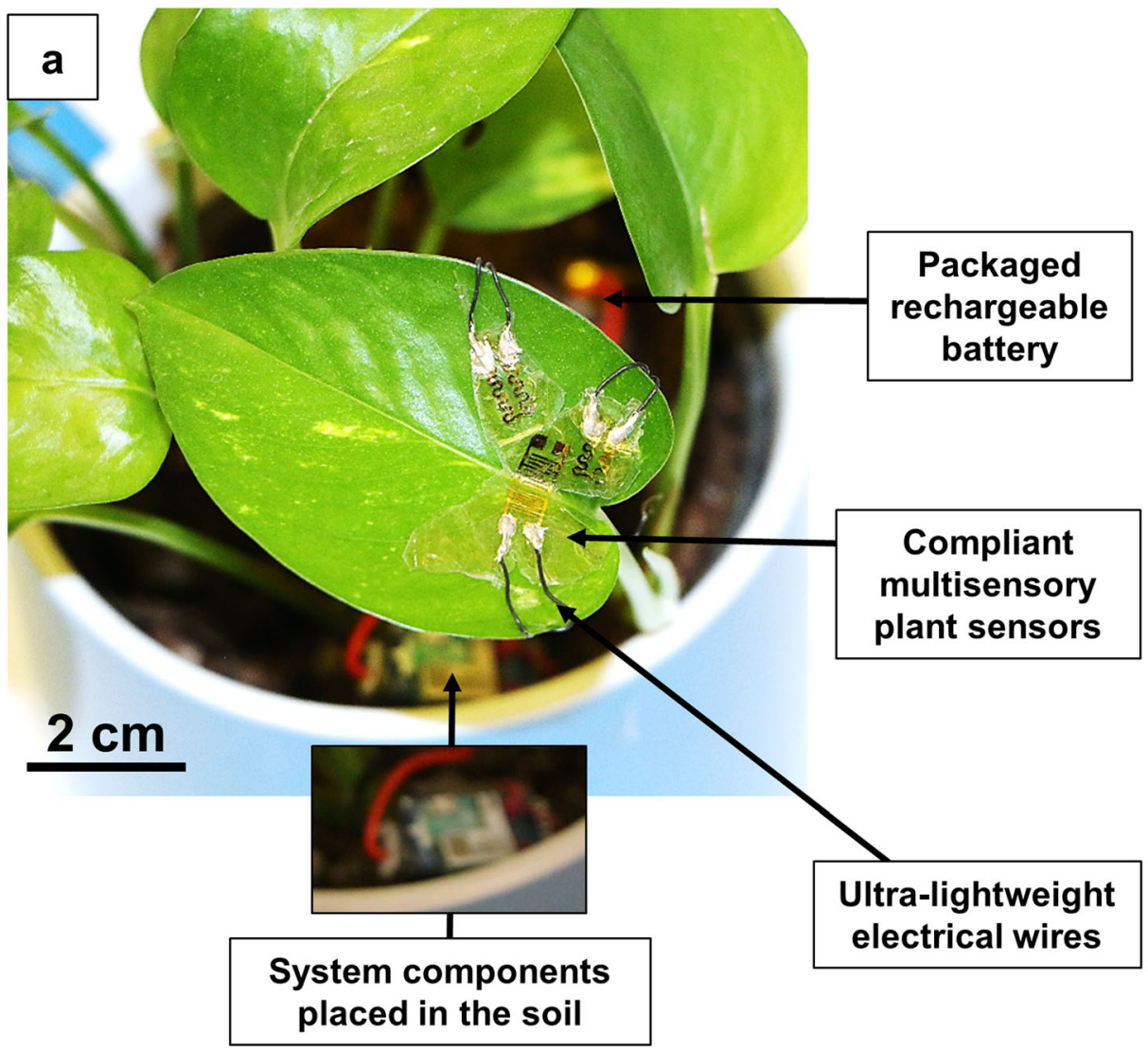

b

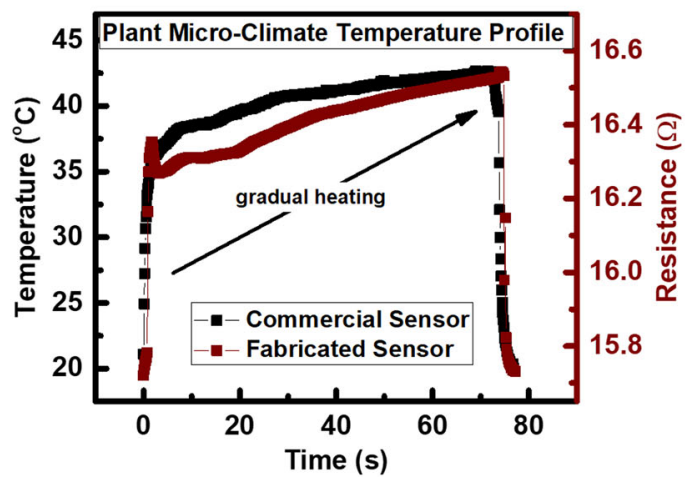

C

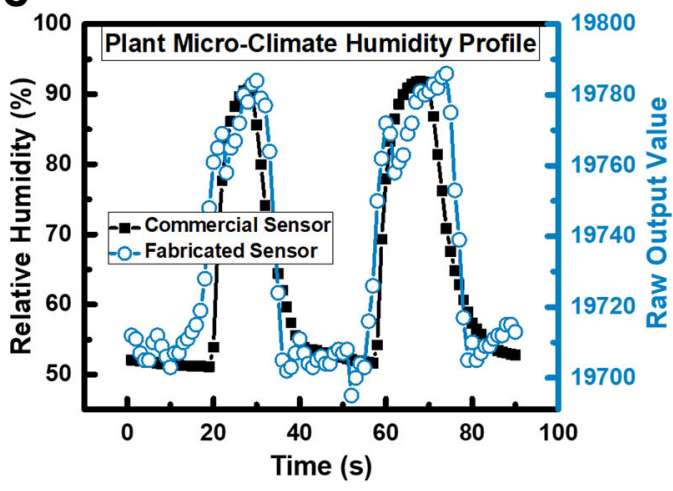

Fig. 4 Autonomous plant wearable for microclimate monitoring. a Digital photograph of the integrated portable system, depicting conformal placement of flexible environmental plant sensors on the surface of plant leaf for real-time monitoring of temperature and humidity levels. Ultra-light electrical wires connect our sensors to the rest of the system. Connected system components (PSoC and rechargeable battery) are then placed on the soil nearby the plant. b Real-time response of fabricated sensor in comparison to commercial temperature sensor, when exposed to local changes in the temperature profile around the plant. c Real-time plot of fabricated humidity sensor in comparison to a commercial sensor's behavior, in response to changes in the humidity levels around the plant

value can be converted into relative humidity by performing a calibration, similar to what we did for temperature sensor (Fig. S1).

Overall, the fabricated sensors have desired linear responses, and display relatively good sensitivities to monitor the necessary microclimate changes around the plant. Temperature sensitivity compares well with the literature, with reported values ranging from $0.0018 /{ }^{\circ} \mathrm{C}$ up to $0.0061 /{ }^{\circ} \mathrm{C} .{ }^{24}$ Likewise, humidity sensor displays high sensitivity compared to flexible polyimide-based humidity sensors, with reported sensitivities as low as $0.0038 / \%$ $\mathrm{RH}^{25}$ and $0.0025 / \% \mathrm{RH}^{16}$ Generally, higher sensitivities can be achieved by optimizing the dimensions of the interdigitated electrodes, such as width, length, and separation between the fingers, as described in the following work. ${ }^{16}$

Plant wearable for local microclimate monitoring

Humidity and temperature levels can influence crop quality and plants' growth, and are generally controlled in greenhouses using sophisticated equipment, where humidity can be one of the most difficult environmental factors to locally control around each crop and plant. ${ }^{26-28}$ The microclimate around each plant is influenced by the location of the plant as well as its response to surrounding environmental factors. For example, humidity levels fluctuate with 
changes in surrounding temperature, and plants are constantly transpiring (an outcome depending on local temperature), which hence adds water vapor to the microclimate environment of that plant. ${ }^{29}$ Humid air directly contributes to problems such as foliar and root diseases, slow drying of the growing medium, plant stress, loss of quality, and loss in yields. ${ }^{30}$ If humidity levels are too low, leaves from the lower part of the plant often drop off, growth slows down, and overall health of leaves deteriorates. This being said, local climate control of both temperature and humidity around plants is crucial. A certain minimum level of humidity is required for optimal plant growth, but not to the point where it reaches the dew point. If the temperature is at or below the dew point, the air cannot hold the moisture in air, and condensation occurs, covering and glazing the leaf surfaces. ${ }^{31}$ As a consequence, condensed water on leaves increases disease problems and minimizes water intake by the plant, increasing nutritional problems. The photosynthesis process is also affected by temperature and humidity levels. Photosynthesis is the process of converting carbon dioxide and water in the plant leaves to produce sugars that are used for energy and growth. ${ }^{31}$ When temperature is high and humidity is normal, more stomata will open, letting in carbon dioxide for active photosynthesis. ${ }^{31}$ If the air is excessively dry and the plant is lifeless, the stomatal openings close, thereby reducing photosynthetic activity and ultimately plant growth. Plant growth depends greatly on the conditions that promote optimal photosynthesis, which in turn is a process majorly influenced by the balance between humidity and temperature.
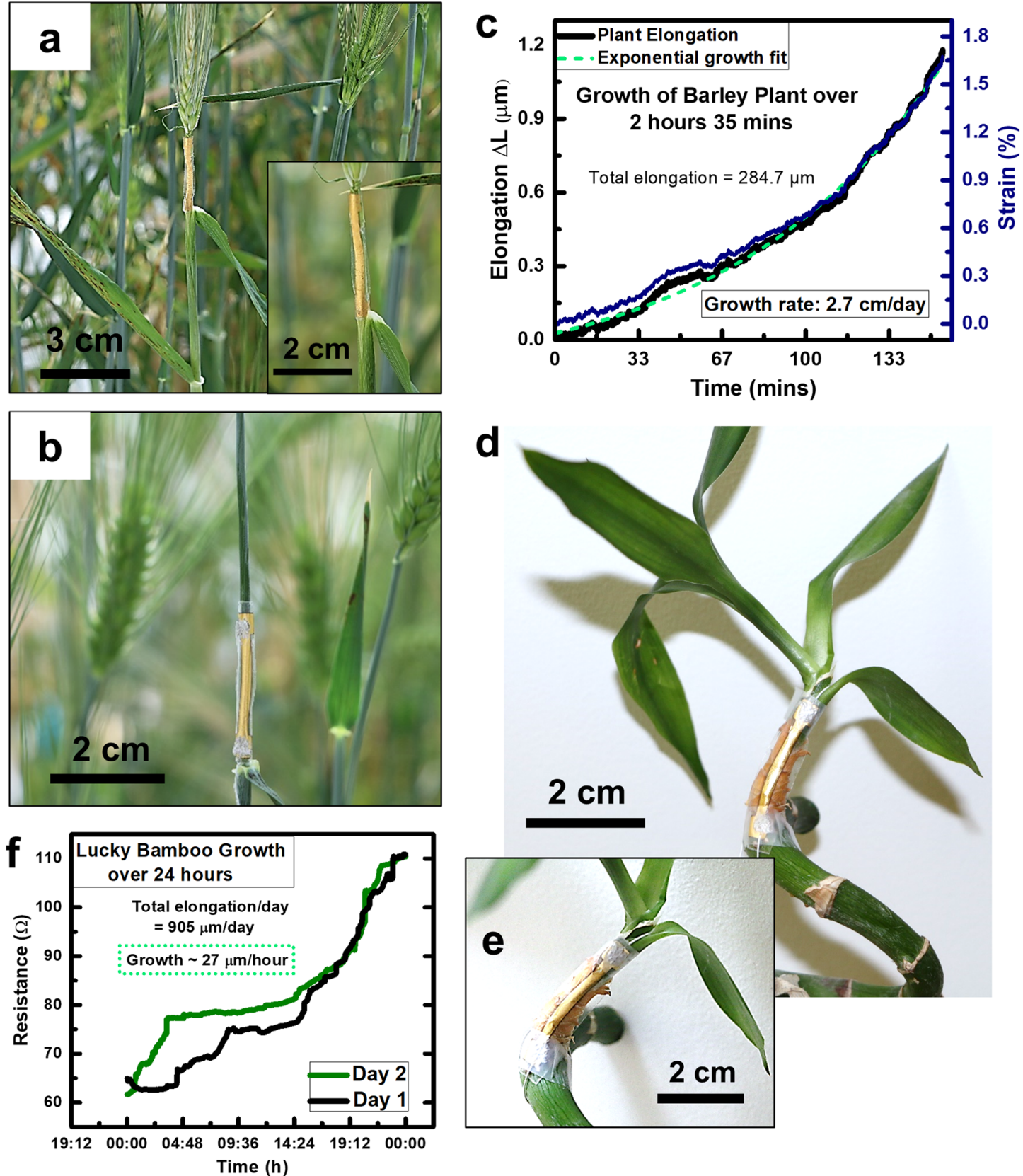

Fig. 5 Quantitative plant growth monitoring. a, b Digital photographs depicting the integration of the strain sensor system on a Barley plant for growth monitoring. c Plot illustrating the Barley plant growth in real time through leaf elongation tracking for a period of $2 \mathrm{~h}$ and $35 \mathrm{~min}$. d, e Digital photographs displaying the seamless integration of the growth strain sensors on the stem of a Lucky Bamboo, in between the internodes. f Quantitative and real-time tracking of Lucky Bamboo growth rate over a period of $24 \mathrm{~h}$ continuously for 2 days 
Consequently, having a wearable device on the plant to provide proper control and continuously monitor the localized microclimate (temperature and humidity simultaneously) around the plants can lead to major increase in the quality and growth rate of plants. Therefore, we develop an environmental plant wearable integrating the butterfly shaped multisensory platform (Figs. $1 \mathrm{~h}$ and $2 \mathrm{a}$ ), aiming at monitoring local temperature and humidity levels. The wearable monitor is deployed by uniformly placing the sensors on the surface of the plant leaf, as depicted in Fig. 4a, then connected to the rest of the system components (PSoC and the battery) (Fig. 2d) using ultra-light electrical wires and silver epoxy. The packaged system components have small footprint and can be seamlessly employed in the soil nearby the plant (Fig. 4a). Realtime monitoring of the plant's microclimate is conducted by wirelessly collecting temperature and humidity data from the developed flexible platform. As means of comparison and support for our findings, data extracted from our autonomous plant system is put in direct comparison with data collected from stateof-the-art commercial sensors (Fig. 4b, c). Figure 4b shows that both the commercial and the fabricated temperature sensors act almost identically in real-time response to local and continuous temperature variations from $\sim 20$ up to $42^{\circ} \mathrm{C}$. Voltage values collected from our plant system were converted to temperature seen by the sensor and then translated to resistance values using sensitivity plot of Fig. 3h and Fig. S1a. The long response profile seen for both the commercial and fabricated sensor is dictated by the continuous and gradual heating in the temperature profile, whereas recovery was almost instantaneous for both sensors since the heating source was immediately removed. Similarly, Fig. 4c shows consistent behavior between fabricated humidity sensor and state-of-the-art commercial sensor. Data from the humidity sensor was collected in real time using our integrated plant system. Steady behavior is shown over two cycles of elevated humidity up to $93 \% \mathrm{RH}$ then recovery down to $42 \% \mathrm{RH}$. Response and recovery profile for both sensors are in close agreement, demonstrating that our humidity sensor compares well to the dependable and commonly used commercial solid-state sensors.

\section{Plant wearable to evaluate plant growth rate}

To monitor plants' growth, researchers currently rely on imaging techniques where they take images of the plant on a daily basis, and use a tailored algorithm to estimate the growth based on leaves shape and structure. ${ }^{32}$ This method helps in recovering the structure of a plant directly from a small set of widely spaced images, however it does not provide quantitative information about the micrometer-level growth rate of the plant in study. Generally, if we look at a plant, it is composed of the shoot system and the root system. The shoot system is the one we see above the ground, and includes organs such as leaves, stems, flowers, and fruits in the primary stages of a plant growth. The functions of the shoot system, present underground, include photosynthesis, reproduction, storage, transport, and hormone production. ${ }^{14}$ In plants, the formation of new cells (signaling growth), tissues, and organs is restricted almost entirely to regions known as meristems. There are three types of meristems, and they are usually located at the tips of stems and roots, between the tip and base of stems and leaves, and sides of stems and roots. The function of these meristems is growth, and increase of length at (i) tips, (ii) between nodes, and (iii) diameter. However, there is a dermal tissue that covers the plant body, consisting of an epidermis made of parenchyma cells in a single layer. ${ }^{14}$ This epidermis on stem and leaves prevents water loss by transpiration and produces a waxy material called cuticle. Therefore, the extension of plant cells responsible for plant growth cannot be monitored from the outside surface of stems and leaves. Growth happens from within the stems and extension is pushed from bottom up, which is reflected through the internal extension of secondary stems, without the local extension of the first stem epidermis.

Therefore, to provide continuous monitoring of plant growth in a low cost yet reliable manner, our approach is to develop a plant wearable that integrates stretchable strain sensors. Unlike the previously discussed methods, this technique allows for a universal and precise quantitative measurement of plant growth characterized by length extension between stem sections. We show proof-of-concept application by implementing our plant wearable on two different types of plants: (i) "Barley Plants" grown in the Greenhouse facility at KAUST, and (ii) "Lucky Bamboo" shoots. Strain sensor is placed in such a way to monitor plant elongation along the stems, which is one of the main indicators of plant growth. Appropriate placement of the sensor depends on the plant species. For Barley plant, most above-ground tissues initially develop from the crown. The first leaf grows upward within the cylinder of the coleoptile, and emerges above the soil, and new leaves emerge in succession up through the sheaths of the older leaves. Barley produces several additional secondary stems termed tillers that emerge up from the crown beginning a few weeks after the emergence of the main stem. ${ }^{33}$ Therefore, Barley used in this experiment were at least 14 days old before we initiated any experiment, to allow some tillering to happen. Tillering stage happens just before stem and flag leaf elongation is possible. Accordingly, the sensor is placed in between two nodes. The first anchor is placed on the main shoot/stem, and the second anchor on the flag leaf in process of elongation, as shown in Fig. $5 \mathrm{a}, \mathrm{b}$. Growth is hereafter reflected through the vertical extension of the flag leaf with respect to the stem. As for the lucky bamboo, it has intercalary meristems located above the bases of leaves and stems, which is a zone of elongation. Hence, the sensor was simply placed on the base of the internode to monitor length extension, as shown in Fig. 5d, e. While strain sensors are placed on the areas of interest, the corresponding integrated system runs down via lightweight electrical wires, similar to the system integration shown in Fig. 4a, and is placed on the soil to forbid additional weight exertion that can prohibit the natural growth and elongation of the plant.

Real-time measurements on the Barley plant were conducted continuously for $<3 \mathrm{~h}$, sufficient time to see micrometer elongation reflecting growth rate. Results in Fig. $5 \mathrm{c}$ show a total leaf elongation of $284.7 \mu \mathrm{m}$ for a total time of $2 \mathrm{~h} 35 \mathrm{~min}$ (with sampling time of $\Delta t=3 \mathrm{~s}$ ), translated into a $1.6 \%$ strain seen by the sensor. The growth seems to have an exponential profile, however on a small scale, assuming constant growth rate throughout the day, plant's growth rate would be approximated to $2.7 \mathrm{~cm} /$ day. This growth was measured while the Barley plant was in optimized environmental conditions in the greenhouse. As for the Lucky bamboo plant, we conduct the experiment over $24 \mathrm{~h}$ for 2 days as seen in Fig. 5 f. Again, we see the same exponential growth behavior. Aside from the minor shifts in initial elongation rates, we see consistency in the growth rate profile over the 2 separate days. Each day induces an increase of $\Delta R=47.67 \Omega$ in the sensor's resistance, translating into a total stem elongation of $904.9 \mu \mathrm{m} /$ day. Growth rate of lucky bamboo can differ depending on the growth conditions and environmental parameters. Our lucky bamboo was kept inside an office area while placed in a water vase. Although there are no grounds for growth rate per day comparison, the repeatability of growth behavior over 2 days in a row shows consistency and reliability for plant growth detection.

\section{NATURE-INSPIRED PLANTCOPTER FOR MICROCLIMATE MONITORING IN FIELDS}

For extended applications in large fields, it would be nearly impossible and time consuming to tag each plant with the microclimate monitoring system shown in Fig. 4a. The direct implementation of the sensors on the plant is a useful way for 


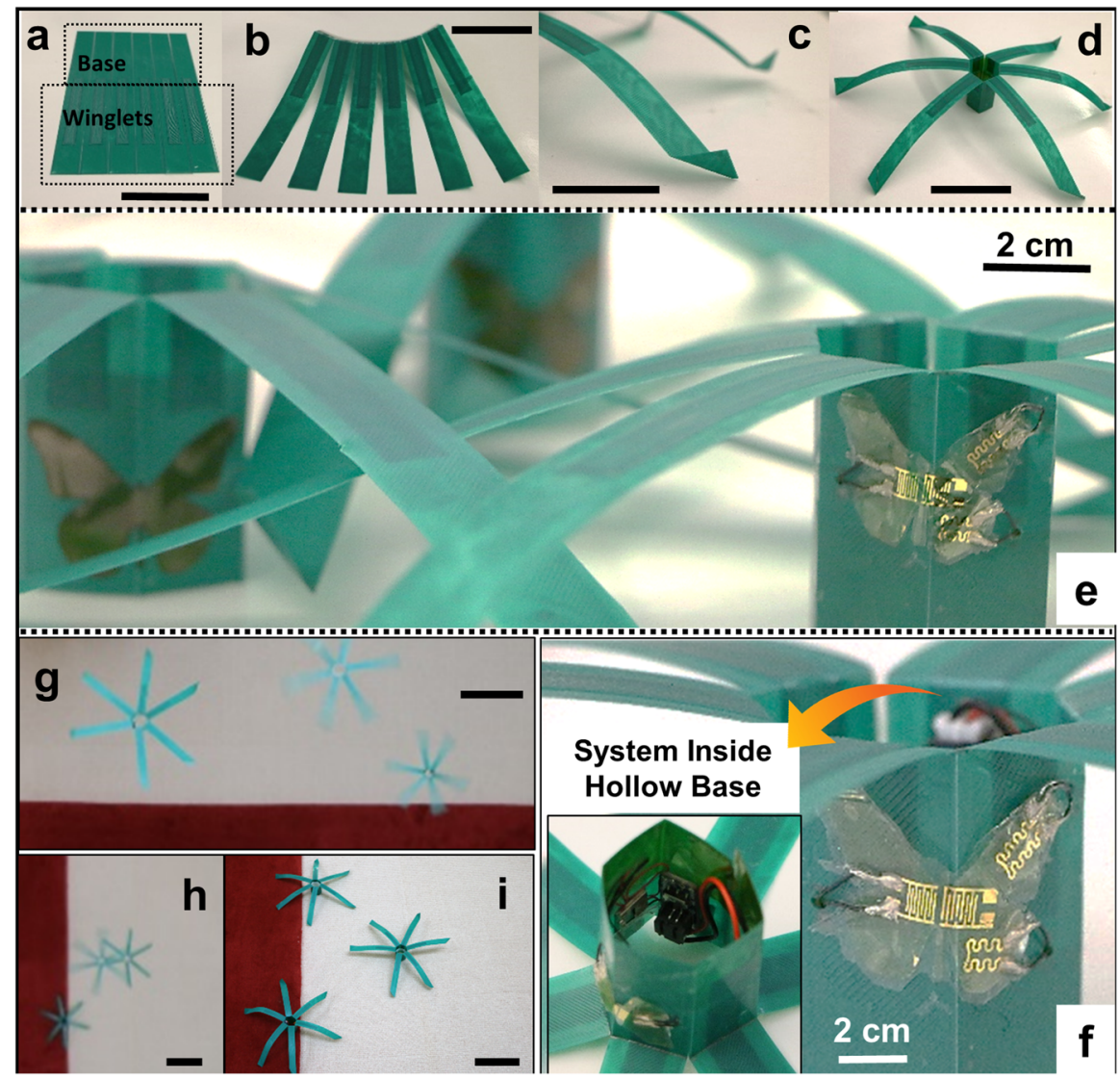

Fig. 6 PlantCopter design for field applications. a-d Sequential photographs of the origami-assembling mechanism adopted to construct the 3D-printed PlantCopter. Scale bar is $6 \mathrm{~cm}$. e Conformal placement of butterfly environmental sensors on the outer shell base of the lightweight PlantCopter. $f$ System components integration within the hollow base of the PlantCopter, providing the necessary balance and center of mass. g-i Digital photographs depicting sequential flight mode of three PlantCopter as they are released in free-fall mode. Scale bar is $10 \mathrm{~cm}$

wide-area monitoring of plants, or for smaller scale studies in greenhouses for ambient conditions optimization. Therefore, we have designed and developed plant sensory system on a passive hovering platform, which we will refer to as a free-fall (gravity is the fuel) "PlantCopter". We envision having thousands of these ultra-lightweight and low-cost plant monitoring copters carried out by a drone. The drone will be then flown over the field and release the copters in mid-air. The optimized design of the copters allows them to rotate and twirl while in free-fall trajectory, exhibiting a smooth flight and dampened landing to not harm the plants or the sensory system. Since we are integrating Bluetooth communication into the wearable, a drone with a master Bluetooth device can sequentially connect to each of the tag in a large field using a unique ID of each Bluetooth chip.

The PlantCopter design was inspired from natural phenomenon we see in our everyday life: the wind-dispersed seeds. Flying maple seeds, also known as double Samaras, have this astonishing winged seed structure defined by two ultra-light winged leafs and separated at the base by a relatively heavier seed. They resemble helicopters in motion as they twirl in free-fall motion to the ground in late summer or early fall. Similarly, the Dandelion flower seeds are known as "helicopter" seeds generally seen floating and spinning through mid-air. They have an ultra-light and fluffy structure, characterized by a fan-like top assembly and connected all the way down to a heavier base. Flying seeds have common structural assemblies, enabling them to twirl and fly in a seamless and stable trajectory: (i) Ultra-light wings/petals assembly: enables flight and swirling motion through a generated lifting force. The geometry, dimensions, and weight of the winglets are interdependent, and affect majorly the resultant lifting force. The dependency of the lift force " $L$ " can be described by the following equation $^{34}$ :

$L=C_{\mathrm{L}} \times \frac{\rho \times v^{2}}{2} \times A$

Where " $C_{\mathrm{L}}$ " is the lift coefficient which is an experiment number containing all complex dependencies, " $\rho$ " is the density of air, " $v^{2 \prime}$ is the square of the air's velocity, " $A$ " is the surface area over which the air is flowing, in this case the helicopter seeds. Shape of the body and its inclination to the flow. Generally, the dependence on body shape, inclination, air viscosity, and compressibility is very complex. $^{34}$ Therefore, for the purpose of our study, we will focus on two main criteria to adjust our lifting force: (1) Surface area-where a greater surface area (width $\times$ length) of the copter wings leads to increased lifting force, and (2) Weight of the Copter-obviously, for the object to fly, the lifting force must be higher than the weight " $W$ " of the copter, which is dictated gravitational the gravitational pull " $g$ " and the mass " $m$ " of the copter, by: $F_{\mathrm{g}}=m \times g$. Therefore, using more lightweight materials will enable us to have enough lifting force without the need to increase the size of our copter.

(ii) A slightly heavier base-center of mass: means for balance, control, and stabilization of the trajectory. This base is crucial 
to maintain steady flight, even through windy days. Without this relatively heavier base, the seeds/copters would be diverting from their original path, flying in a random fashion, and twirling upside down.

(iii) Bended winglets/petals: the petals or wings are slightly bent upward at a shifted angle. This allows to generate a smaller vortex around the winglet tips, which aids in reducing drag force. The result is a desired increase in lifting force and span for a more efficient and smooth flight. The shifted angle incorporated in the bent winglet allows for more twirling during motion.

Incorporating the desired design rules, we created our own dandelion seed inspired PlantCopter design using 3D printing technology as it is a low-cost, robust, and a scalable approach. Design details, 3D printing, and step-by-step assembly with system integration are illustrated in Fig. 6 and discussed in the "Materials and Methods" section. Movie S3 shows the PlantCopter as it swirls down in free-fall motion. The PlantCopter is almost floating as it's spinning down. The flight is very directional, stable, with a very smooth landing (Fig. 6g-i). Furthermore, the demonstration in Movie S4 shows how the PlantCopter can be wirelessly interfaced to our smartphone via Bluetooth. The video shows the PlantCopter being launched from a two-story high building. Before releasing the copter, we first connect to the smartphone via Bluetooth while selecting the option to display reading from the temperature sensors. After the setup, we see the temperature being displayed on the smartphone $\left(26^{\circ} \mathrm{C}\right.$ shown next to the "HEX" tab on the smartphone). Now the copter is released and its trajectory can be observed in the video, showing smooth landing on the ground. As the PlantCopter swirls down in free fall, the Bluetooth connection was not lost, highlighting how the system stays completely intact after the fall. The phone still shows the temperature output from the copter even from a height of $\sim 50$ feet. In the later part of the video, we walk down all the way close to the copter on the ground to show that the system is still functioning properly. This is vital if these copters are to be launched in a large field for climate monitoring. A drone can release the copter in different sections of the field, and the soft landing of our PlantCopter ensures that the system remains intact during launch and landing. Then, the drone can be used to log values from all the PlantCopters even from a height of 50 feet. Furthermore, in Movie S5, we demonstrate the stability of our PlantCopter when it is released outdoors in a windy environment. Three PlantCopters were released in mid-air, and display great balance and steadiness throughout the free-fall trajectory. Even when there is wind, PlantCopters show to be stable and smoothly complete their landing on the ground without losing equilibrium and flipping.

This proof-of-concept demonstration shows great potential for future implementation of PlantCopters in agriculture fields, using the integrated temperature and humidity sensors to continuously monitor the plants' microclimate conditions, collect data wirelessly via a receiver on a drone above the field, and accordingly assess optimized and local irrigation measurements. PlantCopters are made of biocompatible and environmental friendly and recyclable materials. When the end cycle for their usage is reached, we envision the copters to be automatically recollected from the field through an autonomous drone, and can be recycled for future use. Self-driven drones can maneuver themselves, avoid obstacles, identify objects, and easily collect and store the PlantCopters in an integrated compartment. Over time, this vision provides a costeffective approach for monitoring and maintaining plants in large fields.

\section{CONCLUSION}

To understand how the surrounding environment and optimization actions are directly affecting the health of plants, there is an increasing need for portable and remote techniques to provide continuous and quantitative measurement of plant growth rate. In this work, we successfully develop wearable growth and environmental monitors for plants, enabled through ultra-light and compliant sensors design, and coupled with a compact, low power, and BLE-enabled electronic interface. The environmental plant wearable continuously monitors the local humidity and temperature levels from the surface of the plant's leaf, while stretchable strain sensors quantitatively monitor plant growth with micrometer-level detection of elongation. A 3D-printed origami-based PlantCopter design is also demonstrated for revolutionizing plant wearables in large fields. Using plant wearables to evaluate the relationship between the microclimate surrounding each plant and growth rate can open doors to enhanced plant science investigations and optimized agriculture. And the current demonstration can be optimized further from many aspects. Botanists will better understand the growth needs of each individual plant and perform local optimization actions to increase their yields in suboptimal and harsh environments.

\section{MATERIALS AND METHODS}

\section{Stretchable strain sensors process flow}

The fabrication started with preparation of a prestretched PDMS substrate (Fig. 1a, b), followed by straight wires patterning (for the sensor design) by a metal sputtering deposition tool using a shadow mask (Fig. 1c). We start with a PET substrate on top of which we spin coat $100 \mu \mathrm{m}$ PDMS and cure it at $60^{\circ} \mathrm{C}$ for $1 \mathrm{~h}$. After curing, we cut thin PDMS strips of $35 \mathrm{~mm}$ length and $5 \mathrm{~mm}$ width and simply peel them off from the substrate. PDMS strips are then laterally prestrained by $20 \%$ tensile stretch and placed onto another carrier wafer. $\mathrm{O}_{2}$ plasma is then performed at low RF power of $60 \mathrm{~W}$ for $3 \mathrm{~min}$. This acts as a surface treatment for the PDMS and converts it temporarily to a hydrophilic material, and will promote improved adhesion and interface between the PDMS and the metal film. This step is crucial to avoid peeling or delamination of the metal to be deposited. Then, we pattern our strain sensor design of $25 \mathrm{~mm}$ length and $3 \mathrm{~mm}$ width using a shadow mask and sputtering $10 \mathrm{~nm} / 180 \mathrm{~nm}$ of Ti/Au as our main metal film.

After sensor patterning, ultra-lightweight electrical wires are bonded onto the contact pads using silver epoxy. While still prestrained onto the carrier wafer, another layer of PDMS $(100 \mu \mathrm{m})$ is spin coated and cured at $60^{\circ} \mathrm{C}$ for $1 \mathrm{~h}$. Finally, while the sensor was still prestrained, it was packaged using a second layer of 100- $\mu$ m-thick PDMS (Fig. 1d). The sandwiching of the metal between two layers of PDMS leads to improved control and stability of the sensor over numerous cycling of stretch and release. Top PDMS encapsulation puts our active material (metal film) in the neutral plane of the structure and ensures electrical stability by reducing stresses and uniform distribution of strain in the metal film when the sensor is stretched. This enables repeatability of our measurements with reduced hysteresis and drift so we can reuse the sensor. Finally, the sensors are released from the substrate and the metal decompresses back with the PDMS, leading to the buckling phenomena perceived.

After strain release, the PDMS substrate goes back to its original position and the metal film will compress to a smaller length by forming buckles (Fig. 1e). Releasing the prestrained substrates and the residual stress of substrates leads to buckling in the surface topography. These buckles allow the metal film to see less strain and withstand higher stretching before total discontinuity. This process has two advantages: (i) residual stress release from substrates and (ii) increased theoretical strain of routings. The observed wrinkly behavior indeed improves stretchability, but also releasing residual stress from substrates can improve the quality of the metal film. Because of the different thermal expansion coefficients of wires and substrates, the surface topography of the sputtered metal film has two types: wrinkled (compressive stress) or flat (tensile stress). Macroscopically, wrinkles distribute untidily along the prestretched axis, and Y-shaped wire cracks may occur during sputtering, even when mild heating is used during the processes. Microscopically, the buckled sample is continuous and has a grain-like structure, but the flat sample has randomly arranged micro-cracks. ${ }^{15}$ 
Flexible environmental sensors

Materials and structure. Temperature and humidity sensors were integrated on a flexible 4- $\mu$ m-thick polyimide (PI) film (Fig. 1h). Temperature sensor consists of simply patterning a resistive temperature detector (RTD) design using thin metal film of Ti/Au $(10 \mathrm{~nm} / 180 \mathrm{~nm}$ ) (Figs. 1h-4 and 5). Au was used since it has best conductivity and flexibility among commonly used metals. It was also chosen for its noble properties since temperature and humidity sensors are not packaged from the top. Additionally, Au has lower elastic modulus compared to alternatives like copper, which allows it to undergo more bending cycles. Using the same mask and process steps, we pattern humidity sensors, consisting of interdigitated capacitive structures of Ti/Au on PI (Fig. 1h-5). PI substrate will act as the sensing material of the humidity sensor, where dielectric permittivity of $\mathrm{PI}$ increases due to water molecules adsorbed on its surface as moisture levels increase in air. Finally, the PI butterfly shaped platform is released from the Si (100) carrier wafer by simply peeling off the structure from the $\mathrm{Si}$ wafer (Fig. 1h-6), as the adhesion between polyimides and silicon is extremely poor. ${ }^{35}$ Since plants transpire and they can give off water vapor through pores in their leaves, we wanted to isolate this effect so that it does not interfere with our environmental humidity detection. Therefore, we transfer the multisensory platform onto a thin layer of PDMS $(50 \mu \mathrm{m})$, as shown in Fig. $1 \mathrm{~h}-6$. The transfer needs to be done while PDMS is still half cured to ensure proper adhesion between both materials, then we continue the second half curing process of PDMS after the transfer. After transfer, the PDMS can be either manually reshaped into the butterfly shape, or laser patterned for a more scalable approach.

Process flow. We begin by spin coating $4 \mu \mathrm{m} \mathrm{PI}$ on $\mathrm{Si}(100)$ wafer and cure it at $350^{\circ} \mathrm{C} .200 \mathrm{~nm}$ of $\mathrm{Al}$ is sputtered to act as a hard mask to pattern $\mathrm{PI}$ substrate. After lithography step, we etch Al using RIE, followed by another RIE using a mix of $\mathrm{O}_{2}$ and $\mathrm{CF}_{4}$ to cleanly pattern the PI substrate into various shapes of butterflies. Once done, the photoresist would have been completely removed during the $\mathrm{Pl}$ etch process, and thus we move directly onto removing the rest of the Al using aluminum wet etchant solution. The $\mathrm{PI}$ surface is then treated with $\mathrm{O}_{2}$ plasma for $2 \mathrm{~min}$ at $60 \mathrm{~W}$ power to ensure improved adhesion with the subsequent metal deposition step. We sputter $10 \mathrm{~nm} / 180 \mathrm{~nm} \mathrm{Ti} / \mathrm{Au}$, followed by lithography and RIE etch to pattern both humidity and temperature sensors designs. Finally, the integrated sensors die is easily peeled off from the Si wafer. On the side, we prepare $50-\mu \mathrm{m}$ thick PDMS spin coated on a PET wafer. We half cure it at $60^{\circ} \mathrm{C}$ for $30 \mathrm{~min}$ and transfer the released butterfly sensory platform onto the PDMS and leave it to continue curing for another $30 \mathrm{~min}$ at $60^{\circ} \mathrm{C}$. This ensures high bonding at the PI/PDMS interface. Finally, the PDMS is repatterned into the desired butterfly shape, either manually using a scalpel or using laser patterning for a more automated and scalable approach.

\section{Temperature sensor experimental setup}

Temperature sensor is tested using a thermal chuck probe station, allowing us to heat the sensor's surroundings from 10 to $32^{\circ} \mathrm{C}$ with steps of $0.2{ }^{\circ} \mathrm{C}$. Corresponding resistance values were collected using a semiconductor analyzer, which provides better resolution for detecting small resistance changes corresponding to $\Delta T=0.2{ }^{\circ} \mathrm{C}$ temperature variations. Real temperature seen by the sensor (i.e., experienced by the metal film) is identified using a thermocouple attached to the surface of the sensor's electrode. This calibration is important to ensure appropriate sensitivity determination.

\section{D-printed plant copter and origami assembly}

We chose ABS (acrylonitrile butadiene styrene) over PLA (polylactic acid or polylactide) mainly because it has a much lower density of $\rho_{\mathrm{ABS}}=1.04 \mathrm{~g} /$ $\mathrm{cm}^{3}$ in comparison to $\rho_{\mathrm{PLA}}=1.23-1.25 \mathrm{~g} / \mathrm{cm}^{336}$, hence will result in higher lifting force with fixed copter dimensions. The PlantCopter design incorporates a fan-like top structure with six winglets and a hollow base in which the system will be incorporated to act as the center of mass (Fig. 6). To achieve the lightest weight possible from the ABS material, the thickness of the structure should be made out of only one printed ABS layer. Due to printing restrictions in filament-based 3D printers, we could not print only one layer in the $z$-direction, and hence the base would be thicker. As an alternative, we decided to adopt an origami assembly, as shown in the sequential figures of Fig. $6 a-d$. We print a $2 D$ sheet of $A B S$ with dimensions $L \times W=17 \mathrm{~cm} \times 12 \mathrm{~cm}$ (Fig. 6a). The total length is sectioned in a way that $5 \mathrm{~cm}$ will serve as the height of the base, while the rest of the $12 \mathrm{~cm}$ will correspond to the length of each winglet. The total width of the sheet corresponds to six sections of $2 \mathrm{~cm}$ width each, equivalent to one winglet. As can be also noticed from Fig. 6a, we print halfway through, a second layer of ABS $(5 \times 1 \mathrm{~cm})$ on each winglet section. This serves as extra support for keeping the winglets bent at a straighter angle in the last stages of the assembly. Next step consists of taking scissors and simply cutting through the sheet to form the winglets as shown in Fig. 6b. Third step, the edges of each winglet are bent with a slight $45^{\circ}$ angle twist, at about $1 \mathrm{~cm}$ from the winglet tip (Fig. $6 \mathrm{c}$ ). The last stage consists of slight bending of the sheet to define each section and shape the final assembly of the base and winglets, as shown in Fig. $6 \mathrm{~d}$. The plant sensors wearable is finally placed on the outer surface of the PlantCopter as depicted in Fig. 6e, where the compact system is integrated from within the hollow base, in such a way to provide the necessary balance and symmetrical weight for the center of mass (Fig. 6f).

\section{DATA AVAILABILITY}

The data that support the findings of this study are available from the authors on reasonable request. The authors declare that the data supporting the findings of this study are available within the article and the corresponding Supplementary Information File.

\section{ACKNOWLEDGEMENTS}

We thank Professor Mark Tester and his lab members from the Salt Lab and Plant Sciences Program in the Biological and Environmental Sciences and Engineering (BESE) division at King Abdullah University of Science and Technology (KAUST), for their contribution to grow Barley plants for this project. This publication is based on the work supported by the King Abdullah University of Science and Technology (KAUST) Office of Sponsored Research (OSR) under Award No. Sensor Innovation Initiative OSR-2015-Sensors-2707 and KAUST-KFUPM Special Initiative OSR-2016-KKI2880.

\section{AUTHOR CONTRIBUTIONS}

MMH conceptualized the idea and directed the study. JMN fabricated, characterized, and analyzed the data. SMK developed the integrated circuits and systems, characterized, and analyzed the data. JMN and SMK contributed equally to this work. DRV assisted in design of the PlantCopter. MMN assisted with 3D printing. ASA performed wet chemical-based experiments. All authors contributed to writing the manuscript.

\section{ADDITIONAL INFORMATION}

Supplementary information accompanies the paper on the npj Flexible Electronics website (https://doi.org/10.1038/s41528-018-0039-8).

Competing interests: The authors declare no competing interests.

Publisher's note: Springer Nature remains neutral with regard to jurisdictional claims in published maps and institutional affiliations.

\section{REFERENCES}

1. Mortensen, L. M. Effects of air humidity on growth, flowering, keeping quality and water relations of four short-day greenhouse species. Sci. Hort. 86, 299-310 (2000).

2. Sánchez-Blanco, M. J., Ferrández, T., Navarro, A., Bañon, S. \& Alarcón, J. J. Effects of irrigation and air humidity preconditioning on water relations, growth and survival of Rosmarinus officinalis plants during and after transplanting. J. Plant Physiol. 161, 1133-1142 (2004).

3. Körner, O. \& Challa, H. Process-based humidity control regime for greenhouse crops. Comput. Electron. Agric. 39, 173-192 (2003).

4. Zhu, J.-K. Plant salt tolerance. Trends Plant. Sci. 6, 66-71 (2001).

5. Sunkar, R. Plant stress tolerance. Methods Mol. Biol. 639, 401 (2010).

6. Link, P. PlantLink http://myplantlink.com/ (2017).

7. Power, P. F. Parrot Flower Power http://global.parrot.com/au/products/flowerpower/ (2017).

8. Parrot. Parrot Pot https://www.parrot.com/us/connected-garden/parrot-pot\#inthe-box (2017).

9. Trimble Inc. (2017). Active imaging systems for plant growth monitoring. US9582873B2. 
10. James, J. 2016 IEEE Region 10 Humanitarian Technology Conference (R10-HTC) 1-5 (IEEE, Agra, 2016).

11. Meng, R. et al. Growth curve registration for evaluating salinity tolerance in barley. Plant Methods 13, 18 (2017).

12. Tai, W.-C. et al. 2017 International Conference on Applied System Innovation (ICASI) 1130-1133 (IEEE TSSC, Sapporo, 2017).

13. Tang, W., Yan, T., Ping, J., Wu, J. \& Ying, Y. Rapid fabrication of flexible and stretchable strain sensor by chitosan-based water ink for plants growth monitoring. Adv. Mater. Technol. 2, 1700021 (2017).

14. Junction, B. Plant Structure Bi http://www.biologyjunction.com/ plant_structure_bi1.htm (2017).

15. Hocheng, H. \& Chen, C.-M. Design, fabrication and failure analysis of stretchable electrical routings. Sensors 14, 11855-11877 (2014).

16. Lee, C.-Y., Wu, G.-W. \& Hsieh, W.-j Fabrication of micro sensors on a flexible substrate. Sens. Actuators A Phys. 147, 173-176 (2008).

17. Dai, C.-L. A capacitive humidity sensor integrated with micro heater and ring oscillator circuit fabricated by CMOS-MEMS technique. Sens. Actuator B Chem. 122, 375-380 (2007).

18. Tetelin, A., Pellet, C., Laville, C. \& N'Kaoua, G. Fast response humidity sensors for a medical microsystem. Sens. Actuator B Chem. 91, 211-218 (2003).

19. Amjadi, M., Pichitpajongkit, A., Lee, S., Ryu, S. \& Park, I. Highly stretchable and sensitive strain sensor based on silver nanowire-elastomer nanocomposite. ACS Nano 8, 5154-5163 (2014)

20. Kang, D. et al. Ultrasensitive mechanical crack-based sensor inspired by the spider sensory system. Nature 516, 222-226 (2014).

21. Yang, T. et al. Structural engineering of gold thin films with channel cracks for ultrasensitive strain sensing. Mater. Horiz. 3, 248-255 (2016).

22. Yamada, T. et al. A stretchable carbon nanotube strain sensor for human-motion detection. Nat. Nanotechnol. 6, 296-301 (2011).

23. Nassar, J. M., Rojas, J. P., Hussain, A. M. \& Hussain, M. M. From stretchable to reconfigurable inorganic electronics. Extrem. Mech. Lett. 9, 245-268 (2016).

24. Harada, S., Honda, W., Arie, T., Akita, S. \& Takei, K. Fully printed, highly sensitive multifunctional artificial electronic whisker arrays integrated with strain and temperature sensors. ACS Nano 8, 3921-3927 (2014).

25. Zampetti, E. et al. Design and optimization of an ultra thin flexible capacitive humidity sensor. Sens. Actuator B Chem. 143, 302-307 (2009).

26. Paulitz, T. C. \& Bélanger, R. R. Biological control in greenhouse systems. Annu. Rev. Phytopathol. 39, 103-133 (2001).
27. Van Straten, G., Challa, H. \& Buwalda, F. Towards user accepted optimal control of greenhouse climate. Comput. Electron. Agric. 26, 221-238 (2000).

28. Dole, J. M. \& Wilkins, H. F. Floriculture: Principles and Species (Prentice-Hall Inc., Upper Saddle River, NJ, 1999).

29. Shelton, S. Greenhouse Product News. Sweating High Humidity. https://gpnmag. com/article/sweating-high-humidity/ (2005).

30. Horticulture, P. T. How Does Humidity Influence Crop Quality? http://www. pthorticulture.com/en/training-center/how-does-humidity-influence-cropquality/ (2016).

31. Pro-Mix. How Does Humidity Influence Crop Quality? http://www.pthorticulture. com/en/training-center/how-does-humidity-influence-crop-quality/ (2016).

32. Ward, B. et al. European Conference on Computer Vision 215-230 (Springer, Zurich, 2014).

33. Anderson, P. M., Oelke, E. A. \& Simmons, S. R. Growth and Development Guide for Spring Barley https://www.extension.umn.edu/agriculture/small-grains/growthand-development/spring-barley/\#fig10 (2013).

34. NASA. The Lift Equation https://www.grc.nasa.gov/www/k-12/airplane/lifteq.html (2015).

35. Jou, J. H., Liu, C. H., Liu, J. M. \& King, J. S. Adhesion of polyimide to silicon and polyimide. J. Appl. Polym. Sci. 47, 1219-1232 (1993).

36. BotFeeder. ABS and PLA Comparison Table https://www.botfeeder.ca/abs-vs-pla/ (2017).

\section{(c) (i)}

Open Access This article is licensed under a Creative Commons Attribution 4.0 International License, which permits use, sharing, adaptation, distribution and reproduction in any medium or format, as long as you give appropriate credit to the original author(s) and the source, provide a link to the Creative Commons license, and indicate if changes were made. The images or other third party material in this article are included in the article's Creative Commons license, unless indicated otherwise in a credit line to the material. If material is not included in the article's Creative Commons license and your intended use is not permitted by statutory regulation or exceeds the permitted use, you will need to obtain permission directly from the copyright holder. To view a copy of this license, visit http://creativecommons. org/licenses/by/4.0/.

(c) The Author(s) 2018 